\title{
AVALIAÇÃO DA IDADE GESTACIONAL DE RECÉM-NASCIDOS PRÉ-TERMO ATRAVÉS DO EXAME NEUROLÓGICO E DAS ESCALAS NEONATAIS E OBSTÉTRICA
}

\author{
Cristiane Egewarth ${ }^{1}$, Fernanda Dias Almeida Pires², Ana Guardiola ${ }^{3}$
}

\begin{abstract}
RESUMO - A mortalidade perinatal e a incidência de sequelas neurológicas em prematuros têm diminuído, dado os avanços da perinatologia. A avaliação acurada da idade gestacional (IG) é um componente essencial para o manejo dessas crianças. Procuramos avaliar o desempenho do teste neurológico em comparação com as escalas obstétrica (DUM) e neonatais (de Dubowitz e de Capurro). e comparar as idades gestacionais obtidas após a aplicação das diferentes escalas. Foram examinados 35 recém-nascidos, sendo excluídos aqueles com qualquer comorbidade. Após a aplicação das escalas e determinação das idades gestacionais, realizou-se o exame neurológico e anamnese com os pais. Os resultados obtidos demonstraram que o método neurológico de André-Thomas avalia os padrões do exame neurológico clássico do recém-nascido e, embora não seja um método amplamente utilizado na prática médica, mostrou ser fidedigno para a avaliação da IG.
\end{abstract}

PALAVRAS-CHAVE: recém-nascido prematuro, idade gestacional, exame neurológico.

\begin{abstract}
Assessment of gestational age of preterm newborn based on neurological examination, obstetric dating criteria and pediatrics criteria

ABSTRACT - Perinatal mortality and the incidence of neurological sequelae in preterm infants have diminished owing to advances in perinatology. The accurate assessment of gestational age is a very important component of the medical practice and for the newborn's management. Herein we provide a study to evaluate the neurological examination in comparison to the obstetrics dating criteria and pediatrics scales (Dubowitz and Capurro) to determine the gestational age as well as to compare the gestational age obtained with the different methods. We studied 35 preterm newborns leaving out those ones with any pathologies. The different scales to determine the gestational age were administered to each infant, beyond the neurological examination. We conclude that André-Thomas method is an appropriate clinical tool for reliable determining for gestational age in preterm newborns.
\end{abstract}

KEY WORDS: preterm newborn, gestational age, neurological examination.

Estima-se que anualmente, no mundo, 13 milhões de crianças nasçam prematuras ${ }^{1}$. Define-se como parto pré-termo aquele cuja gestação termina entre a $20^{\mathrm{a}}$ e a $37^{a}$ semanas $^{2}$. A avaliação acurada da idade gestacional (IG) é componente essencial de uma boa prática tanto obstétrica quanto neonatal. Muitas técnicas para estimativa da IG estão disponíveis. A data da última menstruação (DUM) é utilizada na obstetrícia, sobretudo para determinar a data provável do parto². A IG neonatal, segundo Dubowitz, é determinada pela inspeção dos diversos sinais físicos e características neurológicas que variam conforme a idade e a maturidade fetais ${ }^{3}$. Os critérios físicos que amadurecem com o avançar da idade fetal incluem firmeza progressiva do pavilhão da orelha, volume crescente do tecido mamário, diminuição dos pêlos finos e imaturos de lanugem sobre o dorso e diminuição da opacidade da pele. Capurro também utilizou critérios somáticos para a determinação da IG. Tais sinais são avaliados durante o primeiro dia de vida e lhes são atribuídos pontos. Um valor cumulativo correlaciona-se com a IG e essa correlação geralmente é precisa, com margem de erro de 2 semanas ${ }^{4}$.

Disciplina de Neurologia do Departamento de Neurologia e Neurocirurgia da Fundação Faculdade Federal de Ciências Médicas de Porto Alegre (FFFCMPA), Porto alegre RS, Brasil: ${ }^{1}$ Acadêmica do $6^{\circ}$ ano do curso de Medicina da FFFCMPA; ${ }^{2}$ Médica Residente do Serviço de Neurologia do Complexo Hospitalar da Santa Casa de Porto Alegre; ${ }^{3}$ Livre Docente em Neurologia Infantil, Professora Adjunta de Neurologia da FFFCMPA. Estudo realizado com o apoio CNPq - PIBIC.

Recebido 23 Janeiro 2002, recebido na forma final 3 Maio 2002. Aceito 17 Maio 2002.

Dra. Ana Guardiola - Departamento de Neurologia e Neurocirurgia, FFFCMPA - Rua Sarmento Leite 245 - $90050-140$ Porto Alegre RS Brasil. FAX: 5132148181. 
A mortalidade perinatal e a incidência de sequelas neurológicas em crianças prematuras têm diminuído, dados os avanços da perinatologiaa, ${ }^{5,6}$. Mesmo as crianças de muito baixo peso ao nascer têm melhores chances de sobrevivência e estão menos sujeitas à elevada incidência de severas sequelas ${ }^{5,7,8}$. Entretanto, as crianças nascidas pré-termo parecem estar mais propensas a disfunções cognitivas na escola e a distúrbios de comportamento ${ }^{5,7,9}$. Salientase a importância da identificação do desenvolvimento anormal de uma criança o mais precocemente possível, para que qualquer distúrbio secundário possa ser prevenido. É difícil, porém, identificar padrões alterados do desenvolvimento precocemente, pois não há concordância sobre o desenvolvimento neurológico normal dos prematuros 5 .

Thomas ${ }^{10}$ e Dargassies ${ }^{11}$ demonstraram que os recém-nascidos (RN) têm um comportamento neurológico semelhante para uma mesma idade gestacional, atingido o termo in ou ex-útero. Para isso, utilizaram a técnica de exame idealizado por André-Thomas, avaliando 100 crianças prematuras, com idades gestacionais variando entre 28 e 37 semanas. Determinaram as "etapas evolutivas das idades-chave", conforme as características maturativas neurológicas. Outros autores têm encontrado diferenças em várias funções, dentre elas: postura, controle da cabeça, reflexo de Moro e orientação visual, sugerindo a ocorrência de influências pós-natais ${ }^{5}$.

Prechtl e Beintema ${ }^{12}$ foram os primeiros a utilizar os estados comportamentais como parte integrante da avaliação neurológica do RN a termo, mostrando a importância da sua relação com vários reflexos do RN. São eles: estado 1 (sono quieto): olhos fechados, respiração regular, sem movimentos corpóreos; estado 2 (sono ativo): olhos fechados, respiração irregular, podendo ocorrer movimentos corpóreos; estado 3 (despertar quieto): olhos abertos, respiração regular, sem movimentos; estado 4 (despertar ativo): olhos abertos, respiração irregular, movimentos corpóreos presentes. estado 5 (choro): criança chorando.

Certos reflexos mostram dependência nítida com alguns estados comportamentais. Os reflexos proprioceptivos são obtidos nos estados 1 e 3, porém encontram-se ausentes ou hipoativos no 2 . Já os exteroceptivos são obtidos nos 2 e 3, porém estão deprimidos no estado 1 . Os reflexos nociceptivos estão presentes em qualquer estado comportamental em que sejam testados ${ }^{13}$. Além disso, o clono do pé que está ausente num RN normal no estado de despertar, pode ser obtido no estado 1 sem que seja patológico.

Os estudos de Minkowski ${ }^{14}$ esquematizaram a existência de nove períodos da maturação da mo- tilidade. McGraw ${ }^{15}$ estabeleceu a existência de quatro fases no desenvolvimento sensório-motor da conduta. O estágio maturativo em que se encontra o sistema nervoso (SN) do RN de termo, com o SN periférico efetivamente mielinizado e várias regiões do SN central (SNC) em processo inicial de mielinização, corresponderá a diversas peculiaridades semióticas, sensitivas, associativas e motoras, que constituem um padrão neurológico possível de ser avaliado pelo exame clínico, que é a base do exame da criança ${ }^{16}$. Esse padrão mostra, em caso de se tratar de prematuro, o quanto ainda lhe falta evoluir para chegar ao padrão do RN de termo e, no lactente, assinala o padrão de maturação neurológica ${ }^{17,18}$.

Neste estudo avaliar a IG utilizando os critérios da escala de André-Thomas, a escala obstétrica (DUM) e as neonatais (de Capurro e de Dubowitz). Comparamos as diferentes idades gestacionais obtidas após aplicação das escalas neurológica, obstétrica e neonatais.

\section{MÉTODOS}

Foi realizado um estudo observacional e de delineamento transversal. Foram examinados $35 \mathrm{RN}$ prematuros, no período de junho/2000 a fevereiro/2001. Foram incluídos RN de até 37 semanas de IG, sendo excluídos todos aqueles com patologia neurológica ou outra comorbidade. Os pais ou responsáveis foram consultados sobre a viabilidade da avaliação neurológica e assinaram um termo de consentimento no caso de concordância. Os dados de gravidez, parto, antecedentes pessoais e familiares dos RN foram colocados em um protocolo.

Os neonatos prematuros foram avaliados através do exame neurológico (EN) quanto aos seguintes itens: ciclo sonovigília, estado de consciência, atitude, emissão de sons, movimentação voluntária espontânea, movimentação involuntária espontânea, reflexos miotáticos fásicos, reflexos superficiais, reflexos arcaicos, movimentação automática, nervos cranianos, sensibilidade dolorosa, trofismo, medidas da cabeça, ângulo poplíteo, tono ativo e passivo ${ }^{13}$.

A técnica de André-Thomas baseou-se na avaliação do tono muscular, descrevendo a postura "tono passivo" (repouso) e o "tono ativo". Além disso, utilizou, também, a evolução de alguns reflexos arcaicos no RN (Moro, marcha, voracidade ou pontos cardeais, preensão palmar e extensão cruzada) ${ }^{10,11,13}$.

As idades gestacionais foram, então, determinadas com a aplicação das diferentes escalas: DUM², Dubowitz ${ }^{3}$, Andre-Thomas ${ }^{10,11,13}$ e Capurro ${ }^{4}$. Sabe-se que o método de Capurro não é um método apropriado para avaliar a IG em prematuros; porém, nesta pesquisa, utilizou-se esse método para observar seu desempenho em comparação com as outras escalas.

A equipe de pesquisa foi constituída da médica orientadora do projeto, médica residente do Serviço de Neurologia e da acadêmica do curso de medicina. 
Os RN prematuros foram examinados na Unidade de Tratamento Intensivo ou no Alojamento Conjunto da Maternidade Mário Totta do Complexo Hospitalar da Santa Casa de Porto Alegre pela médica residente do Serviço de Neurologia da Santa Casa e pela acadêmica do curso de medicina. As examinadoras foram treinadas pela coordenadora da pesquisa, de tal forma que as avaliações dos prematuros fossem homogêneas.

As crianças foram avaliadas individualmente em seus próprios berços ou incubadoras. O tempo usado para a avaliação de cada criança foi aproximadamente 45 minutos.

Após codificação, revisão e conferência manual de todos os protocolos, procedeu-se a entrada de dados em banco. As variáveis numéricas foram descritas em média \pm desvio-padrão; as variáveis categóricas, em frequência e proporção. Para armazenamento e manutenção do banco de dados, foi utilizado o programa MS Excel 2000. Utilizou-se o pacote estatístico SPSS (Statistical Package for Social Science 10.0). Para comparação de proporções utilizou-se o teste do qui-quadrado. A comparação entre médias foi feita utilizando-se o teste ANOVA (Analysis of Variance). Nas correlações foi usado o coeficiente de correlação de Pearson bicaudal. O nível de significância assumido foi $5 \%$.

No planejamento do estudo, considerou-se a pesquisa como sendo do grupo com risco mínimo, classificada no grupo II do artigo $9^{\circ}$ da Resolução 011316/88, referendada pela Resolução 196/96 do Conselho Nacional de Saúde do Ministério da Saúde, pois esta inclui somente avaliação neurológica clínica. O projeto foi analisado e aprovado pelo Comitê de Ética em Pesquisa da ISCMPA.

\section{RESULTADOS}

Foram analisados os dados de $35 \mathrm{RN}$, dos quais 21 eram do sexo masculino e 14 do feminino. Trinta e dois RN eram da raça branca e 3 eram da negra.

A idade, em horas de vida, em que os RN foram examinados foi 24 horas; apenas 2 RN foram avaliados com 48 horas de vida.

O peso dos RN variou entre 990 e 3040 gramas, nos meninos e entre 1320 e 2710 gramas nas meninas. A estatura mínima, nos meninos, foi $36 \mathrm{~cm}$ e a máxima foi $49 \mathrm{~cm}$; nas meninas esta variação foi 37 e $50 \mathrm{~cm}$.

Nesta amostra, 15 dos nascimentos foram por parto vaginal, dos quais 8 eram meninos e 7 eram meninas; em 1 menino e em 1 menina o parto foi vaginal com forceps. Nos outros 18 nascimentos 0 parto foi cesáreo, sendo 12 meninos e 6 meninas.

Quando foi feita a adequação do peso do RN à IG, pelas curvas de Battaglia e Lubchenco ${ }^{19}, 20$ foram classificados como adequados para a idade gestacional (AIG), 1 como grande para a idade gestacional (GIG) e 14 como pequenos para a idade gestacional (PIG).

Os dados antropométricos dos RN estão discriminados na Tabela 1, não havendo diferenças estatisticamente significativas em relação ao sexo. As di-
Tabela 1. Características dos recém-nascidos por sexo (valor médio).

\begin{tabular}{lccc}
\hline Características & $\begin{array}{c}\text { Masculino } \\
\mathrm{n}=21\end{array}$ & $\begin{array}{c}\text { Feminino } \\
\mathrm{n}=14\end{array}$ & $\mathrm{p}$ \\
\hline Peso (g) & $1865,7 \pm 590,9$ & $2005,3 \pm 460,7$ & $\mathrm{NS}$ \\
Estatura (cm) & $42,6 \pm 4,1$ & $43,6 \pm 3,7$ & $\mathrm{NS}$ \\
PC (cm) & $30,4 \pm 2,3$ & $31,0 \pm 1,9$ & NS \\
PT (cm) & $27,9 \pm 3,1$ & $28,4 \pm 1,8$ & NS \\
Apgar1 & $6,2 \pm 2,0$ & $5,7 \pm 2,6$ & NS \\
Apgar5 & $7,9 \pm 1,2$ & $8,3 \pm 1,4$ & NS \\
\hline
\end{tabular}

PC, perímetro cefálico; PT, perímetro torácico; NS, diferença estatisticamente não significativa.

Tabela 2. Idade gestacional (em semanas) por raça (valor médio).

\begin{tabular}{llll}
\hline $\begin{array}{l}\text { Método de } \\
\text { determinação da IG }\end{array}$ & Branca & Negra & $p$ \\
\hline DUM & $34,2 \pm 3,6$ & $34,7 \pm 0,5$ & NS \\
André-Thomas & $34,6 \pm 1,9$ & $34,3 \pm 0,7$ & NS \\
Capurro & $34,7 \pm 2,5$ & $36,0 \pm 2,5$ & NS \\
Dubowitz & $29,3 \pm 1,0$ & $29,2 \pm 1,0$ & NS \\
\hline
\end{tabular}

IG, idade gestacional; DUM, data da última menstruação; NS, diferença estatisticamente não significativa.

Tabela 3. Idade gestacional (valor médio, em semanas) e sexo.

\begin{tabular}{lccc}
\hline IG & Masculino & Feminino & $\mathrm{p}$ \\
\hline DUM & $33,0 \pm 3,1$ & $35,9 \pm 3,1$ & $0,013^{*}$ \\
André-Thomas & $34,5 \pm 2,1$ & $34,6 \pm 1,5$ & $\mathrm{NS}$ \\
Capurro & $34,4 \pm 2,6$ & $35,5 \pm 2,3$ & $\mathrm{NS}$ \\
Dubowitz & $29,5 \pm 0,8$ & $28,9 \pm 1,0$ & $\mathrm{NS}$ \\
\hline
\end{tabular}

IG, Idade Gestacional; DUM, Data da Última Menstruação; NS, diferença estatisticamente não significativa; * diferença estatisticamente significativa.

ferentes idades gestacionais não tiveram associação com a raça (Tabela 2).

Na determinação das idades gestacionais utilizando-se os quatro métodos em relação ao sexo, observou-se significância estatística somente quando a IG foi aferida pela DUM. (Tabela 3). Os RN desta pesquisa encontravam-se, pela DUM, entre 26 e 37 semanas; pela escala de Dubowitz, entre 28 e 30; pelo método de André-Thomas, entre 29 e 37; e pelo de Capurro, entre 29 e 37 semanas. Das 35 crianças, 29 tinham a IG avaliada pela ecografia e variavam entre 27 e 37 semanas. 
Tabela 4. Idade gestacional e apresentação.

\begin{tabular}{lccc}
\hline IG & Cefálica & Pélvica & $\mathrm{p}$ \\
\hline DUM & 35,2 & 35,3 & NS \\
Andre Thomas & 34,5 & 34,0 & NS \\
Capurro & 35,4 & 33,4 & NS \\
Dubowitz & 29,8 & 27,9 & $0,048^{*}$ \\
\hline
\end{tabular}

IG, Idade Gestacional; DUM, Data da Última Menstruação; NS, diferença estatisticamente não significativa; *diferença estatisticamente significativa.

Tabela 5. Idade gestacional (valor médio, em semanas) e exame neurológico.

\begin{tabular}{lccc}
\hline IG & \multicolumn{3}{c}{ Exame Neurológico } \\
& Normal & Alterado & $\mathrm{p}$ \\
\hline DUM & $34,7 \pm 3,2$ & $33,4 \pm 3,6$ & $\mathrm{NS}$ \\
André-Thomas & $35,2 \pm 1,4$ & $33,5 \pm 2,0$ & $0,007^{*}$ \\
Capurro & $34,7 \pm 2,7$ & $35,1 \pm 2,2$ & $\mathrm{NS}$ \\
Dubowitz & $29,5 \pm 1,0$ & $28,7 \pm 0,8$ & $\mathrm{NS}$ \\
\hline
\end{tabular}

IG, idade gestacional; DUM, data da última menstruação; NS, diferença estatisticamente não significativa; *diferença estatisticamente significativa.

Tabela 6. Matriz de correlação entre as diferentes idades gestacionais.

\begin{tabular}{lccc}
\hline IG & André-Thomas & Capurro & Dubowitz \\
\hline DUM & $r=0,555^{*}$ & $r=0,479^{*}$ & $r=0,317$ \\
André-Thomas & -- & $r=0,269$ & $r=0,461^{*}$ \\
Capurro & $r=0,269$ & - & $r=0,551^{*}$ \\
Dubowitz & $r=0,461^{*}$ & $r=0,551^{*}$ & -- \\
\hline
\end{tabular}

r, coeficiente de correlação de Pearson; *diferença estatisticamente significativa.

Quando se relacionou a IG com a forma de apresentação cefálica ou pélvica dos RN, observou-se significância estatística apenas quando aquela foi determinada pelo método de Dubowitz; havendo significância entre os mais prematuros e a posição pélvica $(p=0,048)$ (Tabela 4).

A Tabela 5 mostra as relações entre o $\mathrm{EN}$ e as diferentes idades gestacionais, observando-se associação somente quando a IG foi aferida pelo método de André-Thomas.

O Tabela 6 mostra as correlações entre os diferentes métodos de determinação da IG.

\section{DISCUSSÃO}

Behrman e col. salientam que a raça deve ser considerada especialmente significativa, uma vez que as taxas de baixo peso ao nascer para mulheres negras são o dobro das taxas para mulheres brancas². No presente estudo, predominavam as crianças brancas, na mesma proporção observada para a população em geral, em nosso meio. Não se observou, portanto, associação entre raça e IG.

A avaliação da vitalidade do RN imediatamente após o parto tem sido feita através dos escores numéricos sistematizados por Apgar ${ }^{21}$ que, pela sua praticidade receberam logo aceitação universal. Esses escores não são capazes de avaliar toda a complexidade dos eventos ocorridos com o RN por ocasião do parto e, menos ainda de informar sobre o SN, uma vez que avaliam somente as estruturas do tronco cerebral responsáveis pelo sistema cardiorrespiratório ${ }^{22,23}$. Os escores de Apgar entre os RN desta amostra foram semelhantes, não havendo, portanto, associação com o sexo dos RN.

A avaliação da IG permite a detecção de padrões anormais de crescimento fetal, desse modo auxiliando a predizer as complicações neonatais de lactentes grandes ou pequenos para a IG ${ }^{2}$. Nesta pesquisa, quando a IG foi determinada pela DUM conforme o sexo, observou-se diferença estatisticamente significativa entre os 2 grupos, sendo a média da IG menor no sexo masculino, como previamente relatado na literatura ${ }^{7}$. Tal fato não foi observado quando os outros métodos de determinação da IG foram utilizados.

Pelo método de Dubowitz de determinação da IG, que também avalia as características somáticas dos RN, a apresentação pélvica ocorreu em RN de mais baixa IG quando comparada com a apresentação cefálica, na qual os RN apresentaram maior tempo de gestação, não mostrando significância em relação aos outros métodos de determinação da IG.

O EN do RN baseia-se principalmente no sistema motor, enfatizando tono muscular e reflexos ${ }^{8,13}$. Apesar desse tipo de avaliação ser muito útil na verificação da maturação do SNC e na identificação de danos neurológicos severos, não tem mostrado forte valor preditivo para o êxito neurocomportamental?

Robinson ${ }^{20}$, em estudo prévio com relação aos vários reflexos que podem ser pesquisados nos prematuros, conclui que os reflexos de preensão palmar e plantar, fase extensora do reflexo de Moro, reflexo de retirada à estimulação dolorosa, reflexo de Galant e piscamento à estimulação luminosa, estão sempre presentes, independente de idade gestacional. $\mathrm{Na}$ presente investigação, o EN alterado mostrou relação com a IG, somente quando esta foi determinada pelo método de André-Thomas. O EN mostrou- 
se semelhante entre os sexos. Depreende-se, então, que o método proposto por André-Thomas para aferir a IG é um indicador do perfil neurológico do RN.

A escala de Dubowitz, não é considerada um método acurado de avaliação da IG em RN prematuros com menos de $1500 \mathrm{~g}^{24}$. Porém, os critérios neurológicos associados com os critérios somáticos desta escala mostraram boa acurácia na estimativa da IG, inclusive em prematuros com mais de $1500 \mathrm{~g}^{25}$. Os resultados desta pesquisa mostram que o método de André-Thomas, embora não amplamente utilizado na prática médica, apresentou correlação com o método de Dubowitz e com a DUM, constituindo outro recurso semiológico para a avaliação da IG.

\section{CONCLUSÃO}

Pela análise dos resultados obtidos nesta pesquisa, durante o período de observação, pode-se concluir que: 1 . O gênero dos recém-nascidos prematuros não mostrou diferenças significativas em relação ao peso, estatura, PC, PT e Apgar. 2. Somente houve relação entre IG e sexo quando essa foi aferida pela DUM, observando-se menor IG nos meninos. 3. Foi observado relação entre EN alterado e IG quando essa foi avaliada pelo método de André-Thomas. 4. A escala de André-Thomas mostrou ser um método adequado para determinação da IG em recémnascidos prematuros, quando comparado com as outras formas de avaliação da IG

\section{REFERÊNCIAS}

1. Higby K, Suiter CR. A risk-benefit assessment of therapies for premature labour. Drug Saf 1994;21:35-56.

2. Ramos JGL, Martins-Costa SH, Valério EG, Muller ALL. Nascimento prétermo. In Freitas F, Martins-Costa SH, Ramos JGL, Magalhães JA, et al. Rotinas em obstetrícia. 4.Ed. Porto Alegre: Artes Médicas, 2001:69-80.

3. Dubowitz LMS, Dubowitz V, Goldberg C. Clinical assessment of gestational age in the newborn infant. J Pediatr 1970;771:1-10.

4. Capurro H, Korichzky S, Fonseca O, Caldeiro-Barcia R. A simplified method for diagnosis of gestational age in the newborn enfant. J Pediatr 1978;93:120-122.
5. Forslund M, Bjerre I. Growth and development in preterm infants during the first 18 months. Early Hum Dev 1985;10:201-216.

6. Aylward GP, Hatcher RP, Leavitt LA, et al. Factors affecting neurobehavioral responses of preterm infants at term conceptional age. Child Dev 1984;55:1155-1165.

7. Kurtzberg D, Vaughan HGJ, Daum C, Grellong BA, Albin S, Rotkin L. Neurobehavioral performance of low-birthweight infants at 40 weeks conceptional age: comparison with normal full-term infants. Dev Med Child Neurol 1979;21:590-607.

8. Hagberg B, Hagberg G, Olow I. Gains and hazards of intensive neonatal care: an analysis from Swedish cerebral palsy epidemiology. Dev Med Child Neurol 1982;24:13-19.

9. Peterson BS, Vohr B, Staib LH, et al. Regional brain volume abnormalities and long-term cognitive outcome in preterm infants. JAMA 2000;284:1973-1974.

10. Thomas A, Dargassies SA. Le noveau-né normal. In Études neurologiques sur le nouveau-né et le jeune nourrisson. Paris: Masson, 1952:9-127.

11. Dargassies SA. La maturation neurologique du prématuré. Et Neonat 1955;4:71-122.

12. Prechtl HFR, Beintema D. The neurological examination off the fullterm newborn: Clinics in developmental medicine, 12 London.William Heineman, 1964

13. Gherpelli JLD. Avaliação neurológica do recém-nascido prematuro. In Diament AJ, Cypel S. Neurologia infantil 3.Ed. São Paulo: Atheneu, 1996;22-32.

14. Minkowski M. Acerca de la evolución anatomofisiologica de las funcionais cerebrales en el lactente párvulo. In Neuropsicologia y pediatria. Buenos Aires: Alfa, 1956.

15. McGraw MB. The neuromuscular maturation of the human infant Reprint edition. New York: Hafner, 1963.

16. Lefèvre AFB. Contribuição para a padronização do exame neurológico do recém-nascido normal. Faculdade de Medicina de São Paulo. São Paulo, FMUSP. 1950.

17. Herschkowitz N. Brain development in fetus, neonate and infant. Biol Neonat 1988;54:1-19.

18. Rotta NT. Problemas neurológicos do recém-nascido: o exame neurológico. In Miura E (ed.) Neonatologia: princípios e prática. Porto Alegre: Artes Médicas, 1991;305-307.

19. Battaglia FC, Lubchenco LO. A pratical classification of newborn infants by weight and gestational age. Pediatrics 1967;71:159-163.

20. Robinson RJ. Assessment of gestational age by neurological examination. Arch Dis Child 1966;41:437.

21. Apgar V. A porposal for a new method of evaluation of the newborn. Curr Res Anaesth 1953;32:260-267.

22. Dubowitz L, Mercuri E, Dubowitz V. An optimality score for the neurologic examination of the term newborn. J Pediatr 1998;133:406-416.

23. Haddad B, Mercer BM, Livingston JC, Talati A, Sibai BM. Outcome after successful resuscitation of babies born with Apgar scores of 0 at both 1 and 5 minutes. Am J Obstet Gynecol 2000;182:1210-1214.

24. Sanders M, Allen M, Alexander GR, et al. Gestational age assessment in preterm neonates weighing less than 1500 grams. Pediatrics 1991;88:542-546.

25. Molteno C, Grosz P, Wallace P, Jones M. Neurological examination of the preterm and full-term infant at risk for developmental disabilities using the Dubowitz Neurological Assessment. Early Hum Dev 1995; 41:167-176. 